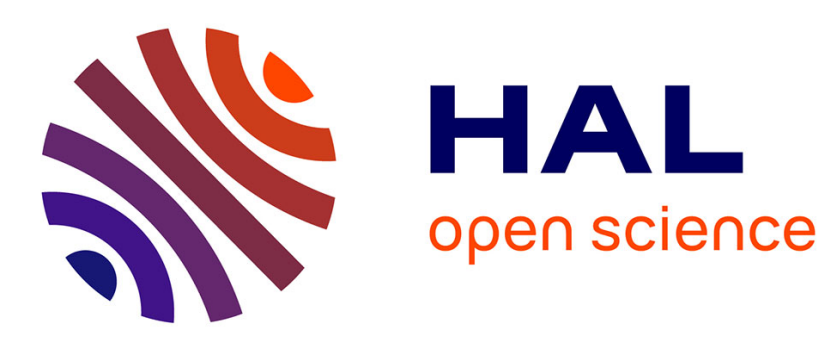

\title{
Application des méthodes de fonction de Green aux défauts dans les semiconducteurs
}

\author{
J.N. Decarpigny
}

\section{To cite this version:}

J.N. Decarpigny. Application des méthodes de fonction de Green aux défauts dans les semiconducteurs. Revue de Physique Appliquée, 1980, 15 (3), pp.661-669. 10.1051/rphysap:01980001503066100 . jpa00244772

\section{HAL Id: jpa-00244772 https://hal.science/jpa-00244772}

Submitted on 1 Jan 1980

HAL is a multi-disciplinary open access archive for the deposit and dissemination of scientific research documents, whether they are published or not. The documents may come from teaching and research institutions in France or abroad, or from public or private research centers.
L'archive ouverte pluridisciplinaire HAL, est destinée au dépôt et à la diffusion de documents scientifiques de niveau recherche, publiés ou non, émanant des établissements d'enseignement et de recherche français ou étrangers, des laboratoires publics ou privés. 


\title{
Application des méthodes de fonction de Green aux défauts dans les semiconducteurs $\left({ }^{*}\right)$
}

\author{
J. N. Decarpigny \\ Laboratoire de Physique des Solides (**), Institut Supérieur d'Electronique du Nord, \\ 3, rue François-Baës, 59046 Lille Cedex, France
}

\begin{abstract}
Résumé. - Parmi les objectifs de l'étude théorique d'un centre profond dans un semiconducteur, la détermination de sa structure électronique et de la reconstruction du réseau cristallin à son voisinage a fait l'objet de multiples approches. Nous nous proposons de décrire celles qui utilisent les méthodes de fonction de Green. Après un rappel des propriétés essentielles de ces fonctions, leur application formelle à la recherche et à la description des états localisés et des résonances est étudiée. La technique est illustrée par le cas de la lacune neutre non relaxée, traitée dans une approximation de liaisons fortes simplifiée, puis par les résultats les plus récents qui permettent notamment la détermination self-consistente du potentiel perturbateur. Ensuite, deux approches voisines de la technique des fonctions de Green sont développées : la méthode des fractions continuées et celle des moments, qui n'utilisent plus les propriétés liées au caractère périodique du cristal parfait, s'apparentant plus aux techniques de clusters. Enfin les diverses approches sont comparées en essayant de dégager les avantages et inconvénients respectifs.
\end{abstract}

\begin{abstract}
Several approaches have been developed for the theoretical study of a deep centre in a semiconductor, and particularly for the determination of its electronic structure and of the lattice distortion in its neighbourhood. This paper describes the approaches which use Green's functions. Following a brief summary of their main properties, a formal application to the study of localized states and resonances is described. The procedure is illustrated by the case of the unrelaxed neutral vacancy, treated at first in the tight-binding approximation and then with more recent formulations which allow a self-consistent determination of the perturbative potential. Then the continued fraction method and the method of moments, which are essentially cluster calculations and do not exploited the translational symmetry of the perfect crystal, are described. At last, the relative merits are compared.
\end{abstract}

1. Introduction. - L'étude de la structure des centres profonds dans les matériaux semi-conducteurs constitue un carrefour de nombreuses techniques expérimentales et théoriques, chacune d'entre elles apportant un élément de solution, mais sans fournir, en général, une image complète et cohérente. Cet état de fait est particulièrement évident dans l'étude théorique de la lacune dans les semi-conducteurs covalents, défaut qui sera l'exemple type choisi dans les diverses sections de cet article. La première approche du problème, due à Coulson, Kearsley [1] et Yamaguchi [2], considère une molécule lacunaire dans laquelle n'interviennent que les quatre premiers voisins du défaut. Elle traite le problème à $n$ électrons et incorpore ainsi les effets de corrélation qui sont trouvés importants. Cette approche a permis le premier calcul de la distorsion Jahn-Teller (Lannoo et Friedel $[3,4]$ ) et l'interprétation probable de la bande d'absorption

$\left({ }^{*}\right)$ Conférence présentée au Congrès de la Société Française de Physique (Toulouse).

$\left({ }^{* *}\right)$ Laboratoire associé au C.N.R.S.
GR1 dans le diamant (Lannoo et Stoneham [5]). Le même modèle de molécule lacunaire mais traité cette fois dans une approximation à un électron, a permis à Watkins $[6,7]$ d'interpréter les spectres RPE de la lacune $V^{+}$dans $\mathrm{Si}$, ainsi que de plusieurs complexes associés. Un article récent de $\mathbf{M}$. Lannoo [8] permet une clarification importante du choix entre techniques à 1 ou $n$ électrons. Autre approche, les méthodes semiempiriques de type Hückel [9] appliquées à des clusters de quelques dizaines d'atomes ont, elles aussi, apporté des résultats intéressants sur la structure électronique de défauts, permettant en particulier le calcul des relaxations et distorsions par effet Jahn-Teller. Ces méthodes qui repèrent difficilement la position des états liés par rapport aux extrêma des bandes ont fait l'objet de critiques sévères $[10,11]$ mais, ces dernières années, des améliorations substantielles [12] ont été apportées (self-consistence, calcul de l'énergie totale...). Plus récemment, une méthode de super réseau, obtenue en répétant périodiquement un cluster de 54 atomes contenant une lacune centrale, a permis l'utilisation d'une technique self-consistente de 
pseudopotentiels [15]. Elle incorpore pleinement, bien sûr, l'effet important de la structure de bandes, mais la taille trop faible du cluster induit une dispersion anormale des états localisés. Enfin, les approches de type Koster-Slater [14] ou fonctions de Green, appliquées dès 1967 au problème de la lacune, en pseudopotentiel par Callaway et al. [15-17] et en liaisons fortes par Lannoo et al. [4, 18, 19] viennent de connaître un nouveau développement. Après avoir permis une résolution précise du problème de la lacune idéale [20] non reconstruite et non self-consistente, elles ont été utilisées pour deux traitements selfconsistents [21, 22] mais toujours sans distorsion. C'est à ces techniques, qui incorporent totalement elles aussi les effets de structure de bandes, que cet article est consacré, son objectif étant de dégager simplement les points essentiels de la méthode et les avantages et inconvénients présentés par ses applications.

L'article est divisé en trois sections principales. La section 2 rappelle le formalisme général, en particulier le lien entre fonction de Green et densité d'états. La section 3 décrit l'approche perturbative. Après avoir dérivé formellement les techniques de recherche des états liés et résonnants et de leurs caractéristiques, plusieurs applications self-consistentes ou non à la lacune non distordue sont développées. Enfin, la section 4 décrit d'autres approches qui évaluent directement la fonction de Green dans la structure perturbée, en insistant particulièrement sur un exemple d'application de la méthode des fractions continuées.

2. Quelques propriétés générales des fonctions de Green. - $H$ étant l'hamiltonien à un électron du système étudié, l'opérateur de Green $G$ est défini par :

$$
G=\lim _{\eta \rightarrow 0^{+}} \frac{1}{E-H+i \eta}
$$

$\mathrm{Si}|k\rangle$ et $\left|k^{\prime}\right\rangle$ représentent deux vecteurs propres de $H$ :

$$
\left\langle k|G| k^{\prime}\right\rangle=\delta_{k, k^{\prime}} \cdot \lim _{\eta \rightarrow 0^{+}} \frac{1}{E-E_{k}+i \eta}
$$

où $E_{k}$ est la valeur propre associée à $|k\rangle$.

De ce fait $G$ peut être redéfini à l'aide des projecteurs par :

$$
G=\lim _{\eta \rightarrow 0^{+}} \sum_{k} \frac{|k\rangle\langle k|}{E-E_{k}+i \eta} .
$$

Une propriété très intéressante de l'opérateur de Green dans l'étude de la structure électronique des défauts est que la partie imaginaire de sa trace, notée $\operatorname{Im} \operatorname{Tr} G$, est directement reliée à la densité d'états. En effet :

$$
\operatorname{Tr} G=\sum_{k} \lim _{\eta \rightarrow 0^{+}} \frac{1}{E-E_{k}+i \eta}
$$

d'où :

$$
\operatorname{Im} \operatorname{Tr} G=-\sum_{k} \lim _{\eta \rightarrow 0^{+}} \frac{\eta}{\left(E-E_{k}\right)^{2}+\eta^{2}} .
$$

Le symbole $\delta$ représentant la fonction de Dirac, on montre aisément que :

$$
\lim _{\eta \rightarrow 0^{+}} \frac{\eta}{\left(E-E_{k}\right)^{2}+\eta^{2}}=\pi \delta\left(E-E_{k}\right) .
$$

Dès lors :

$$
\operatorname{Im} \operatorname{Tr} G=-\pi \sum_{k} \delta\left(E-E_{k}\right)
$$

La somme sur $k$ étant la définition de la densité d'états $n(E)$, on a :

$$
n(E)=-\frac{1}{\pi} \operatorname{Im} \operatorname{Tr} G
$$

Cette relation est particulièrement intéressante puisque, la trace de $G$ étant invariante, on pourra déterminer la matrice $G$ dans n'importe quelle représentation.

Une autre expression de la densité d'états, très utile lorsqu'on doit déterminer sa modification par une perturbation, se déduit immédiatement de (8). Partant de l'identité :

$$
\frac{1}{E-E_{k}+i \eta}=-\frac{\mathrm{d}}{\mathrm{d} E} \log \frac{1}{E-E_{k}+i \eta}
$$

dans une représentation où la matrice de $G$ est diagonale, on a :

$$
\frac{\mathrm{d}}{\mathrm{d} E} \log \operatorname{det} G=-\sum_{k} \frac{1}{E-E_{k}+i \eta}
$$

d'où :

$$
n(E)=\frac{1}{\pi} \frac{\mathrm{d}}{\mathrm{d} E} \operatorname{Im} \log \operatorname{det} G
$$

Enfin, exprimons maintenant $n(E)$ dans une base d'orbitales atomiques $\varphi_{\mathrm{p}}$ où $\mathrm{p}$ peut inclure un indice d'atome (position dans le cristal) et un indice d'état (s ou p par exemple). De (8), on déduit :

$$
n(E)=-\frac{1}{\pi} \operatorname{Im} \sum_{\mathrm{p}}\langle\mathrm{p}|G| \mathrm{p}\rangle .
$$

Utilisant la relation (3) :

$$
n(E)=-\frac{1}{\pi} \operatorname{Im} \sum_{\mathrm{p}, k} \frac{|\langle\mathrm{p} \mid k\rangle|^{2}}{E-E_{k}+i \eta}
$$

puis la relation (6) :

$$
n(E)=\sum_{\mathrm{p}, k}|\langle\mathrm{p} \mid k\rangle|^{2} \delta\left(E-E_{k}\right) .
$$

Définissant :

$$
n_{\mathrm{p}}(E)=\sum_{k}|\langle\mathrm{p} \mid k\rangle|^{2} \delta\left(E-E_{k}\right)
$$


on obtient finalement :

$$
n(E)=\sum_{\mathbf{p}} n_{\mathbf{p}}(E) .
$$

Cette fois, chaque contribution d'un état d'énergie $E_{k}$ à $n_{\mathrm{p}}(E)$ est pondérée par le poids de l'orbitale $\varphi_{\mathrm{p}}$ dans la fonction d'onde de cet état. $n_{\mathrm{p}}(E)$ représente donc la contribution de $\varphi_{\mathrm{p}}$ à la densité d'états totale $n(E)$. Lorsque $\mathrm{p}$ est un indice d'atome, $n_{\mathrm{p}}(E)$ s'appelle une densité d'états locale. C'est une notion qui a été très utilisée dans les études de structure électronique de surfaces car elle permet d'associer certaines caractéristiques de la densité d'états à des positions atomiques.

3. Application aux défauts : traitement perturbatif. - 3.1 Formalisme. - Soit $H_{0}$ l'hamiltonien du cristal parfait, $H$ l'hamiltonien du cristal contenant le défaut, $V$ le potentiel perturbateur dû au défaut. Toute fonction d'onde du cristal avec défaut est solution de :

$$
\left(H_{0}+V\right) \psi=E \psi
$$

soit :

$$
\left(E-H_{0}\right) \psi=V \psi
$$

La solution générale de (17) s'écrira :

$$
\psi=\psi_{0}+G_{0} V \psi
$$

où $\psi_{0}$ est l'onde de Bloch d'énergie $E$, solution générale de l'équation sans second membre, $G_{0}$ l'opérateur de Green du problème non perturbé, $G_{0} V \psi$ une solution particulière de l'équation avec second membre.

Si la valeur propre $E$ du problème perturbé est dans le gap du cristal parfait alors :

$$
\psi=G_{0} V \psi
$$

L'état obtenu constitue un état lié dont l'amplitude s'atténue très rapidement lorsqu'on s'éloigne du centre perturbateur. Décomposant $\psi$ comme combinaison linéaire de fonctions de Wannier ou d'orbitales atomiques par exemple, la relation (19) devient un système homogène d'équations linéaires par rapport aux coefficients du développement. Ce système n'a de solutions non trivialement nulles que si :

$$
\operatorname{det}\left(I-G_{0} V\right)=0 \text {. }
$$

Les solutions de cette équation sont les niveaux d'énergie dans le gap associés aux états localisés. Cette équation montre à l'évidence deux avantages $\mathrm{du}$ traitement perturbatif. Le premier est que la fonction de Green doit être évaluée dans le cristal parfait et non dans le cristal perturbé par le défaut. Le second est que la taille de la partie utile du système d'équations linéaires est fixée par la portée du potentiel qui est toujours très réduite pour un centre profond. Supposons en effet que la base utilisée soit formée d'orbitales localisées sur les sites atomiques. Le potentiel perturbateur $V$ ne couple que quelquesunes d'entre elles et sa matrice s'écrit :

$$
V=\left[\begin{array}{l|l}
\mathbf{V} & 0 \\
\hline 0 & 0
\end{array}\right]
$$

Effectuant la même partition de la matrice $G_{0}$ :

$$
G_{0}=\left[\begin{array}{l|l}
G_{11} & G_{12} \\
\hline G_{21} & G_{22}
\end{array}\right]
$$

on obtient :

$$
\text { dét }\left(I-G_{0} V\right)=\operatorname{dét}\left(I-G_{11} \mathbf{V}\right)
$$

l'ordre du déterminant pouvant être fortement réduit.

Dans le cas où la valeur propre $E$ de l'hamiltonien $H$ du problème perturbé est dans une bande d'énergie permise du cristal la solution la plus générale de (17)

$$
\psi=\psi_{0}+G_{0} V \psi
$$

doit être utilisée. La fonction d'onde $\psi$ n'est plus alors localisée au voisinage du défaut et le phénomène physique représenté est en fait une diffusion des états de Bloch par le potentiel perturbateur. Une information très importante dans ce type de problème est la variation de densité d'états induite dans les bandes. Définissant :

$$
G=\frac{1}{E-H}
$$

on montre facilement que :

$$
G=\frac{1}{E-H_{0}}+\frac{\left(E-H_{0}\right)-\left(E-H_{0}-V\right)}{\left(E-H_{0}\right)\left(E-H_{0}-V\right)}
$$

soit :

$$
G=G_{0}+G_{0} V G
$$

qui est l'équation de Dyson.

Notant $n(E)$ et $n_{0}(E)$ les densités d'états des problèmes perturbé et non perturbé on a, à l'aide de (11)

$$
\delta n(E)=n(E)-n_{0}(E)=\frac{1}{\pi} \operatorname{Im} \frac{\mathrm{d}}{\mathrm{d} E} \log \frac{\operatorname{det} G}{\operatorname{det} G_{0}} .
$$

L'équation de Dyson donnant :

$$
G=G_{0}\left(I-G_{0} V\right)^{-1}
$$

la variation de densité d'états devient :

$$
\delta n(E)=-\frac{1}{\pi} \operatorname{Im} \frac{\mathrm{d}}{\mathrm{d} E} \log \operatorname{det}\left(I-G_{0} V\right)
$$

Définissant le déphasage $\delta(E)$ par :

$$
\delta(E)=-\operatorname{arctg} \frac{\operatorname{Im} \operatorname{det}\left(I-G_{0} V\right)}{\operatorname{Re} \operatorname{det}\left(I-G_{0} V\right)}
$$


on obtient finalement :

$$
\delta n(E)=\frac{1}{\pi} \frac{\mathrm{d} \delta(E)}{\mathrm{d} E} .
$$

L'annulation de Im det $\left(I-G_{0} V\right)$ hors des bandes, puisque $G_{0}$ devient réelle, impose que la variation du déphasage dans une bande soit un multiple de $\pi$. La somme sur les bandes dese variations de $\delta(E)$ est obtenue à l'aide de :

$$
\int \delta n(E) \mathrm{d} E=-N_{1} \pi
$$

où l'intégrale est étendue à toutes les bandes et $N_{1}$ est le nombre d'états dans les gaps. Cette relation (théorème de Levinson) qui assure simplement la conservation du nombre total d'états implique en effet :

$$
\sum_{\text {bandes }} \delta(E)=-N_{1} \cdot \pi
$$

D'autre part, le déphasage prend la valeur $(2 n+1) \frac{\pi}{2}$ où $n$ est un entier quelconque, lorsque :

$$
-\operatorname{Re} \operatorname{det}\left(I-G_{0} V\right)=0 \text {. }
$$

Cette équation est le prolongement de l'équation (20) et ses conséquences physiques peuvent, dans certaines conditions, présenter quelques points communs avec les états liés. Notant $R(E)$ et $I(E)$ les parties réelle et imaginaire de $\operatorname{det}\left(I-G_{0} V\right)$ et $E_{0}$ une solution de (34) on peut développer $I(E)$ et $R(E)$ au voisinage de $E_{0}$, ce qui donne :

$$
\operatorname{tg} \delta(E)=-\frac{I\left(E_{0}\right)+I^{\prime}\left(E_{0}\right)\left(E-E_{0}\right)}{R^{\prime}\left(E_{0}\right)\left(E-E_{0}\right)}
$$

soit, si $R^{\prime} \gg I^{\prime}$ :

$$
\operatorname{tg} \delta(E)=-\frac{I\left(E_{0}\right)}{R^{\prime}\left(E_{0}\right)\left(E-E_{0}\right)} .
$$

Utilisant la relation (31), la variation de densité d'états devient :

$$
\delta n(E)=\frac{\Gamma}{2 \pi} \frac{1}{\left(E-E_{0}\right)^{2}+\frac{\Gamma^{2}}{4}}
$$

avec :

$$
\Gamma=\frac{2 I\left(E_{0}\right)}{R^{\prime}\left(E_{0}\right)}
$$

$n(E)$ peut donc présenter un pic très aigu si la largeur $\Gamma$ est réduite. Cette variation porte le nom de résonance $(\Gamma>0)$ ou d'antirésonance $(\Gamma<0)$. On note qu'une condition d'apparition de tels pics est une variation extrêmement rapide de $R(E)$ au voisinage de $E_{0}$. Une résonance peut dans certains cas s'interpréter comme un élargissement d'un état lié lorsqu'il entre dans une bande d'énergie et interagit avec les fonctions de Bloch. Dans l'espace réel, une autre similitude peut être mise en évidence. $|\alpha\rangle$ désignant des orbitales atomiques (une par atome) imaginons que seul l'élément $\langle 0|V| 0\rangle=V_{0}$ soit différent de 0. Alors :

$$
\begin{array}{r}
\left\langle\alpha\left|G_{0} V\right| \alpha\right\rangle=\sum_{\beta}\left\langle\alpha\left|G_{0}\right| \beta\right\rangle\langle\beta|V| \alpha\rangle= \\
=G_{0}^{00} V_{0} . \delta_{\alpha_{0}} .
\end{array}
$$

D'autre part :

$$
\langle 0 \mid \Psi\rangle=\left\langle 0 \mid \Psi_{0}\right\rangle+\left\langle 0\left|G_{0} V\right| \Psi\right\rangle
$$

soit :

$$
\begin{aligned}
\langle 0 \mid \Psi\rangle & =\left\langle 0 \mid \Psi_{0}\right\rangle+ \\
& +\sum_{\alpha, \beta}\langle 0|G| \alpha\rangle\langle\alpha|V| \beta\rangle\langle\beta \mid \Psi\rangle
\end{aligned}
$$

ou :

$$
\langle 0 \mid \Psi\rangle=\left\langle 0 \mid \Psi_{0}\right\rangle+G_{0}^{00} V_{0}\langle 0 \mid \Psi\rangle .
$$

Finalement :

$$
\frac{|\langle 0 \mid \Psi\rangle|^{2}}{\left|\left\langle 0 \mid \Psi_{0}\right\rangle\right|^{2}}=\frac{1}{\left(1-R_{0}^{00} V_{0}\right)^{2}+\left(I_{0}^{00} V_{0}\right)^{2}} \text {. }
$$

Les équations (34) et (43) montrent qu'à la résonance la contribution de l'orbitale $|0\rangle$ du site perturbé à la fonction d'onde totale peut être très fortement accrue, présentant ainsi une sorte de transition vers la localisation.

Enfin, il faut noter que la connaissance des déphasages détermine directement la variation de la contribution d'une bande à l'énergie électronique totale $\Delta E$ :

$$
\Delta E=\int_{E_{1}}^{L_{2}} \delta n(E) \cdot E \cdot \mathrm{d} E
$$

soit :

$$
\Delta E=\frac{1}{\pi}\left[\left(\delta\left(E_{2}\right)-\delta\left(E_{1}\right)\right)-\int_{E_{1}}^{E_{2}} \delta(E) \cdot \mathrm{d} E\right]
$$

où $E_{1}$ et $E_{2}$ sont les limites de la bande.

Les deux sections suivantes décrivent deux applications de ces techniques à la lacune neutre, non distordue, différant l'une de l'autre par le choix du potentiel perturbateur.

3.2 EXEMPLES D'APPLICATIONS NON SELF-CONSISTENTES A LA LACUNE DANS LES COVAlENTS. - Le traitement décrit ici a été développé par $M$. Lannoo [3, 4] dans un modèle de liaisons fortes très simple et repris plus récemment par $\mathrm{J}$. Bernholc et S. T. Pantélidès [20] à partir d'une meilleure description de la structure de bandes du cristal parfait, mais les deux techniques sont identiques.

Le cristal parfait étant décrit dans une approximation de liaisons fortes utilisant une orbitale $s$ et 3 orbitales p par atome, ou les 4 combinaisons hybridées $\mathrm{sp}^{3}$ correspondantes, la lacune est obtenue en retirant du cristal un atome et ses quatre orbitales sans modifier ni les positions des autres atomes (pas 
de relaxation, pas de distorsion Jahn-Teller) ni les interactions autres que celles qui font intervenir l'atome enlevé (pas de self-consistence). L'hamiltonien du problème non perturbé étant écrit :

$$
H_{0}=\left[\begin{array}{c|c}
H_{\mathrm{x}}^{0} & H_{\mathrm{A}}^{0} \\
\hline\left(H_{\mathrm{A}}^{0}\right)^{T} & H_{\mathrm{B}}^{0}
\end{array}\right]
$$

où $H_{\mathrm{x}}^{0}$ est une matrice $4 \times 4$ correspondant aux orbitales $\alpha$ de l'atome à enlever, l'hamiltonien du problème perturbé est simplement $H_{\mathrm{B}}^{0}$.

Pour utiliser le formalisme précédent, l'hamiltonien $H$ est fabriqué en annulant les termes de couplage dans $H_{\mathrm{A}}^{0}$. Dès lors, les éléments de matrice de potentiel perturbateur sont :

$$
\langle\alpha|V| \beta\rangle=-\left\langle\alpha\left|H_{0}\right| \beta\right\rangle
$$

où $\alpha$ est une des orbitales supprimées. Ceci est bien équivalent à couper les interactions qui connectaient l'atome enlevé au reste du cristal. La taille de la matrice $V$ dépend de la portée des interactions dans le cristal parfait (premiers voisins, seconds voisins...).

Une autre solution pour découpler l'atome enlevé s'avère être plus simple. Si l'on ajoute un même terme $E_{0}$ à chaque élément de la diagonale de $H_{\mathrm{x}}^{0}$ et que l'on fait tendre $E_{0}$ vers l'infini, les couplages dus à $H_{\mathrm{A}}^{0}$ deviennent parfaitement négligeables et les valeurs propres finies de la matrice $H$ ainsi obtenue sont celler de $H_{\mathrm{B}}^{0}$. On réduit ainsi la perturbation à une matrice diagonale $4 \times 4$. De plus, on peut montrer que det $\left(I-G_{0} V\right)$ se factorise en sous-déterminants pour les diverses représentations irréductibles $\mathrm{du}$ groupe de symétrie du potentiel. L'équation des états liés pour la symétrie $A_{1}$ devient :

$$
1-E_{0} G_{\mathrm{ss}}^{0}=0
$$

soit, puisque $E_{0}$ tend vers l'infini :

$$
G_{\mathrm{ss}}^{0}=0 .
$$

De même, pour la symétrie $T_{2}$ :

$$
G_{\mathbf{x}}^{0}=0
$$

la solution obtenue étant 3 fois dégénérée.

Dans les bandes, et pour chaque symétrie, la relation (30) implique :

$$
\delta_{\alpha \alpha}(E)=-\operatorname{Arctg} \frac{E_{0} \operatorname{Im} G_{\alpha \alpha}^{0}}{1-E_{0} \operatorname{Re} G_{\alpha \alpha}^{0}}
$$

soit :

$$
\delta_{\alpha \alpha}(E)=\operatorname{Arctg} \frac{\operatorname{Im} G_{\alpha \alpha}^{0}}{\operatorname{Re} G_{\alpha \alpha}^{0}}
$$

le déphasage total étant :

$$
\delta(E)=\delta_{\mathrm{ss}}(E)+3 \delta_{\mathrm{xx}}(E) .
$$

Les informations essentielles sont ainsi obtenues par le calcul de $G_{\mathrm{ss}}^{0}$ et $G_{\mathrm{xx}}^{0}$.
Notant $|n, \mathbf{k}\rangle$ les états de Bloch et définissant les densités d'états partielles (éq. (14)) :

$$
n_{\alpha \alpha}(E)=\sum_{n, k}|\langle\alpha \mid n, \mathbf{k}\rangle|^{2} \delta\left(E-E_{n, \mathbf{k}}\right)
$$

les relations (3) et (6) donnent :

$$
G_{\alpha \alpha}^{0}(E)=P \int \frac{n_{\alpha \alpha}\left(E^{\prime}\right) \mathrm{d} E^{\prime}}{E-E^{\prime}}-i \pi n_{\alpha \alpha}(E)
$$

où $P$ indique la partie principale. Différentes techniques numériques, Gilat-Raubenheimer [23] par exemple, permettent alors le calcul de la somme (54) sur la zone de Brillouin, puis le calcul de $G$.

Le calcul de $M$. Lannoo utilise pour décrire la structure de bandes le modèle de Leman et Friedel [24] dans lequel les fonctions de Bloch sont construites à l'aide de quatre orbitales hybridées $\mathrm{sp}^{3}$ par atome. Hormis les termes intra-atomiques, ce modèle n'incorpore qu'une intégrale de résonance entre orbitales $\mathrm{sp}^{3}$ d'atomes premiers voisins qui pointent l'une vers l'autre (Fig. 1a). La structure de bandes obtenue donne une description raisonnable des états de valence mais une description trop grossière des états de conduction (Fig. 1b). Les états électroniques de la lacune sont constitués alors essentiellement par les quatre orbitales $\mathrm{sp}^{3}$ pointant vers le site lacunaire (orbitales pendantes). La figure $2 a$ montre que les parties réelles de $G_{\mathrm{ss}}$ et $G_{\mathrm{pp}}$ s'annulent toutes deux dans le gap légèrement au-dessus du milieu du gap, donnant ainsi un état $A_{1}$ et 3 états $T_{2}$ qui sont dégénérés. On peut noter également une antirésonance $A_{1}$ assez aiguë dans le bas de la bande de valence. Le déphasage $\delta_{\text {ss }}$ montre qu'un état est perdu dans la bande de valence, correspondant à un état localisé de même symétrie (Fig. $2 b$ ). Le déphasage $\delta_{\mathrm{pp}}$ montre quant à lui, que l'état de symétrie $T_{2}$ est entièrement perdu par les bandes plates purement $p$ du sommet de la bande de valence. Enfin le calcul des éléments non diagonaux de la fonction de Green du cristal parfait a permis de montrer que $70 \%$ de l'état lié est localisé sur les orbitales pendantes, pour les deux symétries.

Le calcul de Bernholc et Pantélidès utilise pour décrire la structure de bandes le modèle de Pandey et Phillips [25] qui incorpore des interactions jusqu'aux seconds voisins et assure une bonne description de la structure de bandes. L'interprétation des résultats reste qualitativement la même que précédemment mais les positions des niveaux sont modifiées. Dans Si comme dans $\mathrm{Ge}$, il existe un état localisé de symétrie $T_{2}$, trois fois dégénéré, proche du sommet de la bande de valence $(0,27 \mathrm{eV}$ dans $\mathrm{Si}, 0,11 \mathrm{eV}$ dans $\mathrm{Ge}$ ) ainsi que deux résonances $A_{1}$ aiguës. $\mathrm{Ce}$ calcul a, par ailleurs, été appliqué également aux deux lacunes neutres dans GaAs.

3.3 EXEMPLES D'APPLICATIONS SELF-CONSISTENTES a la lacune dans les covalents. - Les deux 


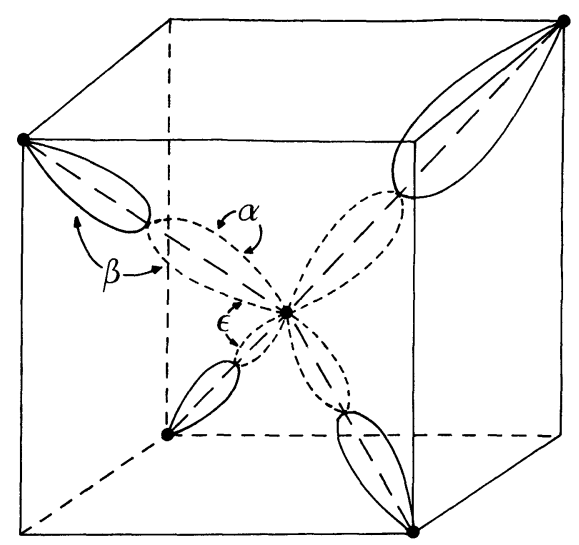

a

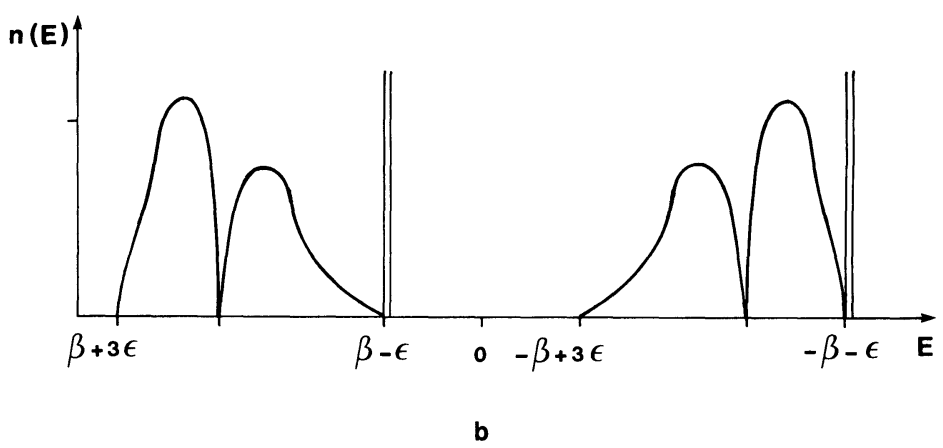

Fig. 1. - Structure de bandes dans le modèle de Leman et Fried el [24] : représentation schématique des interactions (a) et densité d'états (b). [Band structure in the Leman and Friedel's model [24] : schematic representation of the interactions (a) and density of states (b).]
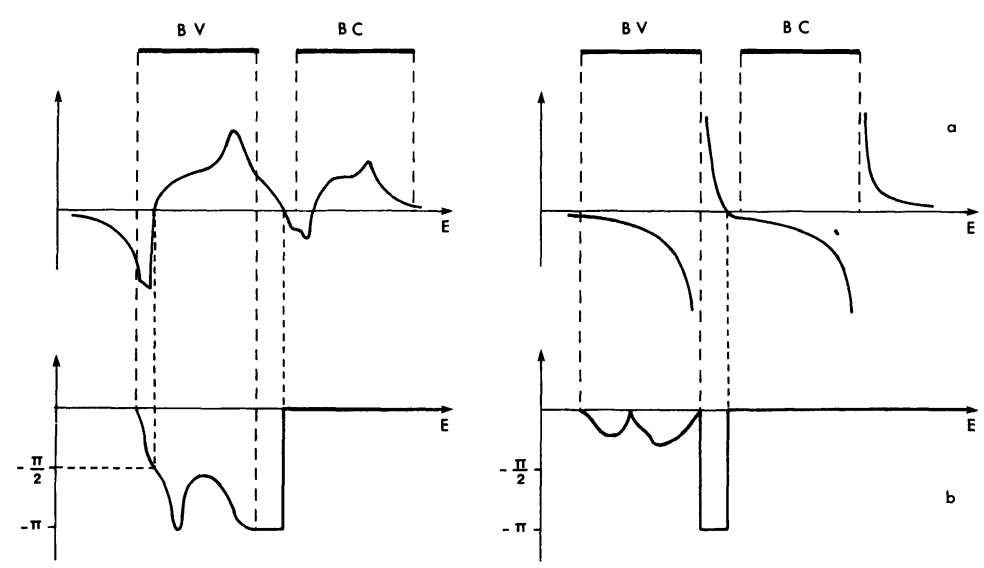

Fig. 2. - Lacune dans le modèle de Lannoo et al. $[4,18]$ : parties réelles de $G_{\mathrm{ss}}$ et $G_{\mathrm{pp}}$ (a) et déphasages $\delta_{\mathrm{ss}}$ et $\delta_{\mathrm{pp}}$ (b).

[Vacancy in the model of Lannoo et al. $[4,18]$ : real parts of $G_{\mathrm{ss}}$ and $G_{\mathrm{pp}}(\mathrm{a})$ and phase shifts $\delta_{\mathrm{ss}}$ and $\delta_{\mathrm{pp}}(\mathrm{b})$.]

travaux décrits ci-dessus admettent que la création d'une lacune ne modifie pas les interactions électroniques autres que celles qui font intervenir l'atome enlevé et, de ce fait, impose le potentiel, comme l'indique la relation (47). A priori, il semble toutefois que même en l'absence de reconstruction la densité électronique de charge puisse se modifier au voisinage du défaut et ceci impose un traitement self-consistent du problème qui produira lui-même le potentiel perturbateur et la nouvelle densité de charge. Suivant cette ligne, deux traitements ont été développés, par Baraff et al. [21] et Bernholc et al. [22] qui aboutissent à des résultats très similaires : état lié $T_{2}$ à $0,7 \mathrm{eV}$ au-dessus de la bande de valence et résonances $\mathrm{A}_{1}$ à environ 1 et $8 \mathrm{eV}$ sous le sommet de la bande de valence, potentiel perturbateur et variation de densité de charge très localisée sur le site lacunaire (Fig. 3). Sans exposer en détail la procédure adoptée, il est intéressant de noter que la méthode employée par Baraff et al. se différencie du forma- 


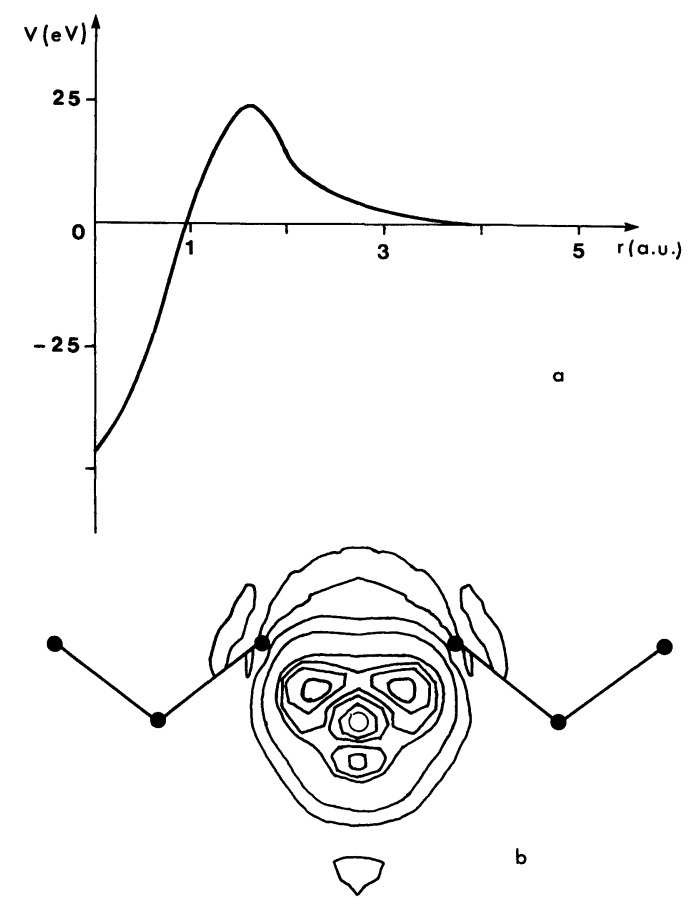

Fig. 3. - Potentiel total self-consistent (a) et variation de la densité électronique de charge dans un plan (110) contenant la lacune [21].

[Total self-consistent potential (a) and variation of the electronic charge density in a (110) plane which contains the vacancy [21].]

lisme général exposé en 3.1 , s'apparentant à la méthode KKR [26].

Définissant (éq. (3)) :

$$
G\left(\mathbf{r}, \mathbf{r}^{\prime}\right)=\sum_{n, \mathbf{k}} \frac{\Psi_{n, \mathbf{k}}(\mathbf{r}) . \Psi_{n, \mathbf{k}}\left(\mathbf{r}^{\prime}\right)}{E-E_{n, \mathbf{k}}}
$$

l'équation (19), caractéristique des états liés, peut se réécrire :

$$
\Psi(\mathbf{r})=\int G\left(\mathbf{r}, \mathbf{r}^{\prime}\right) V\left(\mathbf{r}^{\prime}\right) \Psi\left(\mathbf{r}^{\prime}\right) \mathrm{d}^{3} \mathbf{r}^{\prime}
$$

qui est équivalente à la formulation variationnelle :

$$
\begin{gathered}
\Lambda=\int \Psi^{*}(\mathbf{r}) V(\mathbf{r}) \Psi(\mathbf{r}) \mathrm{d}^{3} \mathbf{r}- \\
-\iint \Psi^{*}(\mathbf{r}) V(\mathbf{r}) G\left(\mathbf{r}, \mathbf{r}^{\prime}\right) V\left(\mathbf{r}^{\prime}\right) \Psi\left(\mathbf{r}^{\prime}\right) \cdot \mathrm{d}^{3} \mathbf{r} \mathrm{d}^{3} \mathbf{r}^{\prime} \\
\delta \Lambda=0 .
\end{gathered}
$$

Utilisant une approximation de type Rayleigh-Ritz, on développe alors $\Psi$ sous la forme :

$$
\Psi=\sum_{i=1}^{n} c_{i} \varphi_{i}
$$

Un extrémum de $\Lambda$ est obtenu si :

$$
\sum_{j=1}^{n} \Lambda_{i j} c_{j}=0 \quad \forall i=1, n
$$

$$
\begin{aligned}
& \text { où : } \\
& \begin{array}{l}
\Lambda_{i j}=\int \varphi_{i}^{*}(\mathbf{r})(\mathbf{r}) \varphi_{j}(\mathbf{r}) \mathrm{d}^{3} r- \\
\quad-\iint \varphi_{i}^{*}(\mathbf{r}) V(\mathbf{r}) G\left(\mathbf{r}, \mathbf{r}^{\prime}\right) V\left(\mathbf{r}^{\prime}\right) \varphi_{j}\left(\mathbf{r}^{\prime}\right) \mathrm{d}^{3} \mathbf{r} \cdot \mathrm{d}^{3} \mathbf{r}^{\prime} .
\end{array}
\end{aligned}
$$

Le système linéaire (60) n'admet de solutions non trivialement nulles que si :

$$
\operatorname{det}\left[\Lambda_{i j}\right]=0
$$

qui devient l'équation des états liés. Cette procédure variationnelle assure que l'erreur sur l'énergie est du second ordre par rapport à l'erreur sur la fonction d'essai. La technique utilisée par Baraff et al. consiste alors à noter que, compte tenu de la forme des relations ci-dessus, il est préférable de rechercher les coefficients $c_{i}$ tels que :

$$
V(\mathbf{r}) \Psi(\mathbf{r}) \simeq V(\mathbf{r}) \sum_{i=1}^{n} c_{i} \varphi_{i}(\mathbf{r})
$$

ce qui autorise un choix de fonctions $\varphi$ localisées seulement dans la région où le potentiel prend des valeurs notables et permet donc une grande flexibilité de la fonction d'essai avec un petit nombre d'orbitales. Les coefficients $c_{i}$ étant obtenus, l'équation (57) permet de remonter à la fonction $\Psi(\mathbf{r})$ totale.

\section{Application aux défauts : méthode des fractions} continuées et méthode des moments. - Plusieurs approximations ont été développées pour calculer la fonction de Green dans des systèmes où la symétrie de translation du cristal parfait est rompue (défauts, surfaces, amorphes, ...) sans faire appel aux techniques perturbatives précédentes. Ces approximations sont en général équivalentes à un développement limité de $G$ suivi d'une resommation. Elles permettent d'atteindre essentiellement la densité d'états totale, les densités d'états partielles ainsi que certaines grandeurs physiques résultant de moyennes sur les états occupés (densité de charge, énergies de cohésion ou de formation, ...).

La méthode des moments consiste à développer la densité d'états partielle :

$$
n_{\mu}(E)=-\frac{1}{\pi} \operatorname{Im} \sum_{n=0}^{\infty} \frac{\left\langle\mu\left|H^{n}\right| \mu\right\rangle}{E^{n}} .
$$

La quantité :

$$
\mu_{n}=\left\langle\mu\left|H^{n}\right| \mu\right\rangle
$$

est appelée moment d'ordre $n$ de la densité $n_{\mu}(E)$ et présente l'avantage de se décomposer sous la forme :

$$
\begin{aligned}
\mu_{n}= & \sum_{\alpha_{1}, \alpha_{2}, \ldots, \alpha_{n-1}}\left\langle\mu|H| \alpha_{1}\right\rangle \times \\
& \times\left\langle\alpha_{1}|H| \alpha_{2}\right\rangle \ldots\left\langle\alpha_{n-1}|H| \mu\right\rangle
\end{aligned}
$$

ce qui, dans les cas simples, revient à un calcul de chemins de $n$ sauts sur le réseau. Cette technique, 
très utilisée dans l'étude des surfaces [27], a été appliquée sous forme simple par M. Lannoo et P. Lenglart [28] aux états liés dans les semiconducteurs.

Une autre méthode, due à Haydock, Heine et Kelly [29] consiste en une tridiagonalisation de l'hamiltonien du système, ce qui revient à écrire la fonction de Green sous forme de fraction continuée. Schématiquement, $|0\rangle$ étant l'orbitale pour laquelle on désire calculer $G_{00}$, on définit les fonctions |1), $\mid 2), \ldots, \mid n), \ldots$ par :

$$
\begin{aligned}
& \mid 1)=|0\rangle \\
& \left.\mid 2)=H \mid 1)-a_{1} \mid 1\right) \\
& \left.\left.\mid 3)=H(2)-a_{2} \mid 2\right)-b_{1} \mid 1\right) \\
& \left.\left.\mid n+1)=H(n)-a_{n} \mid n\right)-b_{n-1} \mid n-1\right) .
\end{aligned}
$$

Les coefficients $a_{i}$ et $b_{i}$, déterminés pour orthogonaliser la fonction $\mid i$ ) aux fonctions d'indices inférieurs sont donnés par :

$$
\begin{aligned}
& a_{i}=\frac{(i|H| i)}{(i \mid i)} \\
& b_{i}=\frac{(i+1 \mid i+1)}{(i \mid i)}
\end{aligned}
$$

Alors, dans la base $\mid i), H$ est bien tridiagonal et $G_{00}$ est obtenu par la relation de récurrence :

$$
G_{n-1, n-1}=\frac{1}{E-a_{n}-b_{n} G_{n, n}}
$$

où :

$$
G_{i, i}=(i|G| i) .
$$

Pour arrêter la récurrence plusieurs techniques sont utilisables. La plus simple consiste à annuler les coefficients $a$ et $b$ au-delà d'un certain niveau $p$, la densité d'états étant alors représentée par $p$ fonctions delta. On peut aussi considérer qu'à partir d'un niveau $p$ les coefficients $a$ et $b$ atteignent des valeurs limites, le reste de la fraction continuée étant alors obtenu analytiquement. Des prescriptions existent permettant le choix de ces valeurs limites. Ce type d'arrêt assure en principe l'exactitude des $2 p$ premiers moments de la densité. Remarquant toutefois que dans des moments d'ordres élevés de nombreux chemins s'enroulent autour de $|0\rangle$ on peut penser améliorer la précision du calcul en utilisant un cluster auquel on impose diverses conditions aux limites : annulation des interactions traversant la surface, imposition de conditions périodiques, raccordement à des réseaux de Bethe (réseaux qui ne présentent pas de boucles), ...

Une application de la méthode de Haydock à la lacune dans le silicium a été développée par Kauffer, Pêcheur et Gerl [30], avec un cluster de 2545 atomes. Leurs paramètres sont obtenus en ajustant à la structure de bandes un hamiltonien effectif comportant des interactions jusqu'aux seconds voisins. Le traitement est, de ce fait, non self-consistent. Leurs résultats, représentés sur les figures $4 a$ et $4 b$ indiquent l'existence d'un état lié $\mathrm{T}_{2}$ à $0,12 \mathrm{eV}$ au-dessus de la bande de valence et d'une résonance $\mathrm{A}_{1}$ à $-0,88 \mathrm{eV}$ dans la bande de valence. On note sur la figure $4 a$ que, compte tenu de la méthode, le gap est une région de densité d'états très faible mais non rigoureusement nulle. De ce fait, sur la figure $4 b$, l'état lié $\mathrm{T}_{2}$ a l'allure d'une résonance très aiguë. Cette méthode a été appliquée par les mêmes auteurs à l'interstitiel dans Si [30], au centre $F$ et aux défauts iso-électroniques dans $\mathrm{ZnS}$ [31], ainsi qu'à une configuration reconstruite de la lacune [32] en vue d'aborder une description de l'effet Jahn-Teller.
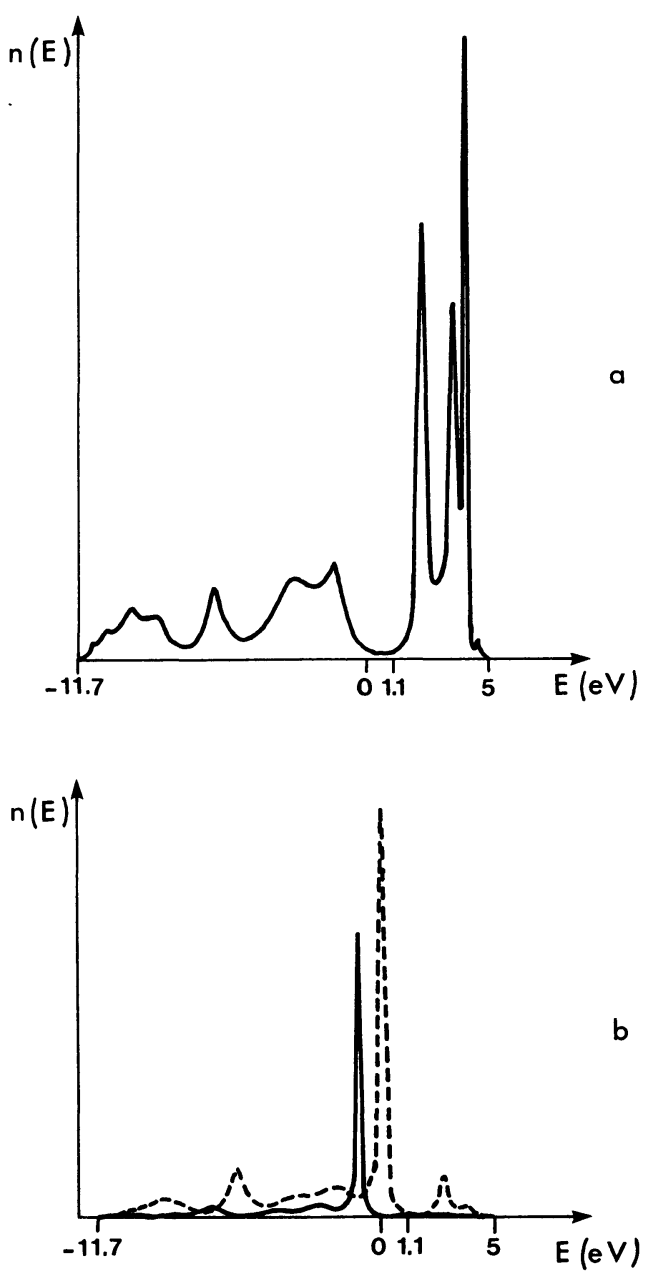

Fig. 4. - Densité d'états du silicium (a) et densité d'états locale sur les premiers voisins de la lacune $\left(-A_{1},-\cdots-T_{2}\right)[30](b)$.

[Silicon density of states (a) and local density of states on the first neighbours of the vacancy $\left(-A_{1},----T_{2}\right)[30](b)$.]

5. Conclusions. - Nous avons essentiellement, dans cet article, exposé divers types d'application de la méthode des fonctions de Green à la lacune et il est intéressant de les comparer, quant à leurs résultats et leurs possibilités. Du point de vue des résultats d'abord, Bernholc et al. ont reproduit, en injectant dans leur méthode perturbative non self-consistente 
(cf. § 3.3) les paramètres de Kauffer et al., l'ensemble des résultats de ces derniers, ce qui peut constituer un test intéressant de leur précision. Du point de vue possibilités, la technique des fractions continuées a déjà été utilisée pour traiter une configuration reconstruite de la lacune. En effet, puisqu'elle n'a jamais recours à la périodicité du cristal parfait, la complexité supplémentaire introduite par cette reconstruction reste faible. Toutefois, le calcul de la fonction de Green doit être fait directement dans la structure perturbée. Au contraire, les méthodes perturbatives n'utilisent que la fonction de Green du cristal parfait dont l'évaluation est plus simple. Ce fait a aussi autorisé les premiers calculs self-consistents puisque dans ces traitements, seuls les éléments de matrice $\mathrm{du}$ potentiel sont à réévaluer à chaque itération. Revenant aux résultats, l'identité de ceux obtenus par Bernholc et al., d'une part, Baraff et al., d'autre part, est un test très positif de leur précision et de leur convergence. On peut penser enfin que ces différentes approches permettront d'atteindre une évaluation correcte de l'énergie et de discuter ainsi de la nature des reconstructions, comme ont commencé à le faire les méthodes du type EHT.

\section{Bibliographie}

[1] Coulson, C. A., Kearsley, M. J., Proc. R. Soc. A 241 (1957) 433.

[2] Yamaguchi, T., J. Phys. Soc. Jpn. 17 (1962) 1359.

[3] Friedel, J., Lannoo, M., Leman, G., Phys. Rev. 164 (1967) 1056.

[4] Lannoo, M., Ann. Phys. 3 (1968) 391.

[5] Lannoo, M., Stoneham, A. M., J. Phys. Chem. Solids 29 (1968) 1987.

[6] WatKINs, G. D., Radiation damage in semiconductors (Paris, Dunod) 1964, p. 97.

[7] Watkins, G. D., Radiation damage and defects in semiconductors (Ed. J. E. Whitehouse, Institute of Physics, London) 1973, p. 228.

[8] Lannoo, M., Defects and radiation effects in semiconductors (ed. J. H. Albany, Institute of Physics, London) 1978. p I

[9] Messmer, R. P., Watkins, G. D., Phys. Rev. B 7 (1973) 2568.

[10] Stoneham, A. M., Theory of defects in solids (Clarendon Press, Oxford) 1975.

[11] Coulson, C. A., Radiation damage and defects in semiconductors (Ed. J. E. Whitehouse, Institute of Physics, London) 1973, p. 249.

[12] Astier, M., Bourgoin, J., 2nd Lund International Conference on deep levels in semiconductors, et Phys. Rev. B 19 (1979) 5265.

[13] Louie, S. G., Schlüter, M., Chelikowski, J. R., Cohen, M. L., Phys. Rev. B 13 (1976) 1654.

[14] Koster, G. F., Slater, J. C., Phys. Rev. 95 (1956) 1167.

[15] Callaway, J., J. Math. Phys. 5 (1964) 783.

[16] Callaway, J., Hughes, A. J., Phys. Rev. 156 (1967) 860 et 164 (1967) 1043.
[17] Callaway, J., Phys. Rev. B 3 (1971) 2556.

[18] Lannoo, M., Lenglart, P., J. Phys. Chem. Solids 30 (1969) 2409.

[19] Djafari-Rouhani, M., Lannoo, M., Lenglart, P., J. Physique 31 (1970) 557.

[20] Bernholc, J., Panteldies, S. T., Phys. Rev. B 18 (1978) 1780.

[21] Baraff, G. A., Schlüter, M., Phys. Rev. Lett. 41 (1978) 892.

[22] Bernholc, J., Lipari, N. O., Pantelides, S. T., Phys. Rev. Lett. 41 (1978) 895.

[23] Gilat, G., Raubenheimer, L. J., Phys. Rev. 144 (1966) 390.

[24] Leman, G., Friedel, J., J. Appl. Phys. Suppl. 33 (1962) 281.

[25] Pandey, K. C., Phillips, J. C., Phys. Rev. Lett. 32 (1974) 1433 et Phys. Rev. B 13 (1976) 750.

[26] Jones, W., March, N. H., Theoretical Solid State Physics, Wiley Interscience, 1 (1973) 541.

[27] Cyrot-Lackmann, F., Adv. Phys. 16 (1967) 393 et J. Phys. Chem. Solids 29 (1968) 1235.

[28] Lannoo, M., Lenglart, P., J. Physique 32 (1971) 427.

[29] Haydock, R., Heine, V., Kelly, M. J., J. Phys. C 5 (1972) 2845 et J. Phys. C 8 (1975) 2591.

[30] Kauffer, E., Pecheur, P., Gerl, M., J. Phys. C 9 (1976) 2319.

[31] Pecheur, P., Kauffer, E., Gerl, M., Phys. Rev. B 14 (1976) 4521.

[32] Kauffer, E., Pecheur, P., Gerl, M., Phys. Rev. B 15 (1977) 4107. 